\title{
Concomitance of Familial Mediterranean Fever and Gitelman syndrome in an adolescent
}

\author{
Bahriye Atmış ${ }^{1}$, Rabia Miray Kışla-Ekinci², Engin Melek ${ }^{1}$, Atıl Bişginn ${ }^{3}$ Mustafa Yılmaz², \\ Ali Anarat ${ }^{1}$, Aysun Karabay-Bayazıt ${ }^{1}$ \\ Departments of ${ }^{1}$ Pediatric Nephrology, ${ }^{2}$ Pediatric Rheumatology and ${ }^{3}$ Medical Genetics, Çukurova University Faculty of \\ Medicine, Adana, Turkey.E-mail: akbayazit@gmail.com
}

Received: 12th January 2018, Revised: 17th May 2018, 30th July 2018, 8th September 2018, Accepted: 17th

September 2018

SUMMARY: Atmış B, Kışla-Ekinci RM, Melek E, Bişgin A, Yılmaz M, Anarat A, Karabay-Bayazıt A. Concomitance of Familial Mediterranean Fever and Gitelman syndrome in an adolescent. Turk J Pediatr 2019; 61: 444-448.

Gitelman syndrome is a renal tubular salt-wasting disorder characterized by hypokalemic metabolic alkalosis with hypomagnesemia and hypocalciuria. Patients occasionally have symptoms in childhood, while diagnosis is often in adulthood. It is inherited by an autosomal recessive manner through SLC12A3 gene mutations. Familial Mediterranean Fever (FMF) is the most common autoinflammatory disorder, inherited by an autosomal recessive manner and characterized by recurrent fever and pleuritis, peritonitis, and synovitis. Mutations in MEditerrenean FeVer (MEFV) gene, coding pyrin protein are responsible for FMF. Both MEFV and SCL12A3 genes were located on chromosome 16. A 9-year-old boy was admitted to our department because of recurrent abdominal pain, fever, joint pain and swelling since he was three years old. He was diagnosed as FMF and MEFV gene sequencing revealed homozygous M694V (c.2080A>G) mutation. At the age of 14 years, polyuria, polydipsia, hypokalemia and mild hypomagnesemia had occurred. Patient was successfully treated with oral supplementation of potassium and magnesium along with colchicine. Molecular genetic analysis including SCL12A3 gene sequencing revealed homozygote IVS4-16G>A (c.602-16G>A) intronic splicing site mutation.

Key words: Gitelman syndrome, Familial Mediterranean Fever, child.

Gitelman syndrome (GS) is a renal tubular saltwasting disorder characterized by hypokalemic metabolic alkalosis with hypomagnesemia and hypocalciuria. It is the most common renal tubular disorder among Caucasians with a prevalence of $1 / 40,000$. Patients occasionally have symptoms in childhood, while diagnosis is often in adulthood. It is inherited by autosomal recessive manner through SLC12A3 gene mutations. ${ }^{1,2}$

Familial Mediterranean Fever (FMF) is the most common autoinflammatory disorder, inherited by an autosomal recessive manner and characterized by recurrent fever and pleuritis, peritonitis, and synovitis. Mutations in MEditerrenean FeVer (MEFV) gene, coding pyrin protein are responsible for FMF. Both MEFV and SCL12A3 genes were located on chromosome $16 .{ }^{3}$ Comorbidity of GS and FMF was previously reported in an adult with heterozygosity for E148Q mutation in MEFV without a confirmed mutation in SLC12A3. ${ }^{4}$ In this report, we present an adolescent diagnosed with FMF in early childhood who went on to developed Gitelman syndrome. 


\section{Case Report}

A 9-year-old boy was admitted to our department because of recurrent abdominal pain, fever, joint pain and swelling since he was three years old. He was born to consanguineous parents. Attacks of abdominal pain and fever in the range of 39 to $40^{\circ} \mathrm{C}$ lasted 24 to 72 hours and repeated 4-5 times a year. During these episodes there were no associated symptoms or other complaints, and between attacks the patient was well. He was diagnosed with FMF since clinical symptoms fulfilled both Yalcinkaya-Ozen and Tel-Hashomer criteria. ${ }^{5,6}$ MEFV gene was sequenced and revealed homozygous M694V (c.2080A>G) mutation. Parental screening showed the heterozygosity for the same mutation. Patient was treated with colchicine at a dose of $1 \mathrm{mg}(0.03 \mathrm{mg} / \mathrm{kg})$ per day and the patient was attack-free for 5 years follow-up with normal serum inflammatory markers such as erythrocyte sedimentation rate (ESR) and $\mathrm{C}$ - reactive protein (CRP) during the attack-free period.

During routine blood analysis in his followup at the age of 14 years, hypokalemia and mild hyponatremia were detected. When he was questioned elaborately for complaints, a history of polydipsia and polyuria for the last six months was stated. Laboratory investigations revealed hypomagnesemia, mild hyponatremia, hypokalemia, a tendency towards metabolic alkalosis with normal serum levels of calcium, phosphate and creatinine. Renal ultrasonography was normal. Twenty-four hour urinary volume was 3000 cc. Fractional excretion of potassium $\left(\mathrm{FeK}^{+}\right)$ was increased and urinary calcium/creatinine ratio was decreased. Fractional excretion of magnesium $\left(\mathrm{FeMg}^{+2}\right)$ was in normal range. Plasma renin concentration was markedly high, whereas plasma aldosterone could not be studied due to technical problems. Laboratory results at the time of diagnosis is given in Table I.

Biochemical triad of metabolic alkalosis, hypomagnesemia and hypocalciuria was suggestive for GS. The patient was successfully treated with oral supplementation of $2 \mathrm{mEq} /$ $\mathrm{kg}$ potassium and $4 \mathrm{mg} / \mathrm{kg}$ magnesium along with colchicine. He had normal serum levels of potassium $(4.01 \mathrm{mmol} / \mathrm{L})$ and magnesium $(1.92 \mathrm{mg} / \mathrm{dL})$ with oral supplementation. Treatment with potassium and magnesium was ceased to rule out that electrolyte abnormalities were not transient. After eight days from cessation of the treatment with potassium and magnesium, our patient had hypokalemia (2.8 $\mathrm{mmol} / \mathrm{L})$, hypomagnesemia $(1.4 \mathrm{mg} / \mathrm{dL})$ and metabolic alkalosis. Oral supplementation of potassium and magnesium were started again. ESR and CRP levels were normal except during the FMF attacks before the diagnosis of GS and also after supplementations for potassium

Table I. Laboratory Results of Our Patient at the Diagnosis of Gitelman syndrome.

\begin{tabular}{|c|c|c|}
\hline Parameter (unit) & Result & Normal range for age \\
\hline Serum sodium $(\mathrm{mmol} / \mathrm{L})$ & 132 & $135-145$ \\
\hline Serum potassium $(\mathrm{mmol} / \mathrm{L})$ & 2.1 & $3.5-5.5$ \\
\hline Serum magnesium (mg/dL) & 1.5 & $1.7-2.4$ \\
\hline Serum bicarbonate (mmol/L) & 28 & $21-26$ \\
\hline Serum calcium $(\mathrm{mg} / \mathrm{dL})$ & 10.5 & $9.6-10.6$ \\
\hline Serum phosphate (mg/dL) & 4.6 & $3.5-4.9$ \\
\hline Serum creatinine $(\mathrm{mg} / \mathrm{dL})$ & 0.5 & $0.8-1.3$ \\
\hline $\mathrm{FeK}^{+}(\%)$ & 22.7 & $4-16$ \\
\hline Urinary $\mathrm{Ca} /$ creatinine ratio $(\mathrm{mg} / \mathrm{mg})$ & 0.037 & $<0.2$ \\
\hline $\mathrm{FeMg}^{+2}(\%)$ & 1.8 & $<4$ \\
\hline Plasma renin $(\mathrm{pg} / \mathrm{ml})$ & $>500$ & $5.41-34.53$ \\
\hline
\end{tabular}

$\mathrm{FeK}^{+}$; Fractional excretion of Potassium.

$\mathrm{FeMg}^{+2}$; Fractional excretion of Magnesium. 
and magnesium.

The patient had one sibling and she was diagnosed FMF with homozygous M694V (c.2080A > G) mutation. Serum electrolytes of the patient's 9-years-old sibling and parents were normal. Follow-up of serum electrolytes in sibling and parents were planned.

In further investigations, next generation sequencing of SCL12A3 gene revealed homozygote IVS4-16G>A (c.602-16G>A) intronic splicing site mutation, that cause frameshift and premature stop codon in mutation tester program. Parental testing also proved the mutation was inherited. The parents were heterozygous for the c.60216G $>$ A mutation. Patient and his parents gave their informed consent prior to the report.

\section{Discussion}

Gitelman syndrome, the most frequent inherited reason of hypomagnesemia is caused by mutations in SLC12A3. The SLC12A3 gene encodes the $\mathrm{Na}+-\mathrm{Cl}-$-cotransporter (NCC) expressing on the apical membrane of the distal collecting tubule (DCT). ${ }^{7}$ Symptoms are generally absent in the first years of life and patients tend to present symptoms towards the end of the first decade. Patients may suffer from a spectrum of hypomagnesemia-related symptoms, including cramps, paresthesia or polyuria, salt craving and thirst. ${ }^{8,9}$ The mechanism of hypomagnesemia in GS is still not fully understood. Some hypotheses include the atrophy of the DCT, a reduced apical membrane potential and a reduction in transient receptor potential melastatin type 6 (TRPM6) activation or mobilization. ${ }^{10}$ Our patient had relevant laboratory findings including mild hypomagnesemia, mild hyponatremia, hypokalemia, increased urinary potassium excretion and hypocalciuria consistent with GS. However, differentiating hypomagnesemia of renal origin from intestinal loss could not been satisfied. Normal FeMg may be due to the fact that prominent hypomagnesemia cause lower filtered load of $\mathrm{Mg}$. Thus, normal or low FeMg does not rule out renal Mg wasting, such as in our patient ${ }^{7}$. Transient electrolyte disturbances were excluded as the patient had recurrent hypokalemia and hypomagnesemia after cessation of supplementation. The other causes of tubulopathies were excluded due to normal urine analysis without glucosuria, proteinuria, hematuria, leukocyturia, normal blood pressure and normal renal function. Also, acute tubulointerstitial nephritis was promptly excluded for these reasons.

Coexistence of GS and FMF has not yet been reported in childhood. ${ }^{11-13}$ Erten et al. ${ }^{4}$ reported the coexistence of GS and FMF in a 46-year-old man with heterozygosity for E148Q mutation in MEFV without a confirmed mutation in SLC12A3. This patient was diagnosed with GS based on clinical and biochemical findings. Improvement of biochemical abnormalities with potassium and magnesium supplementations resulted in clinical improvement at the end of the follow-up of two years in their patient. ${ }^{4}$ Our patient had clinical and laboratory features of FMF and GS, also homozygous mutations in both M694V and SLC12A3 genes. The clinical symptoms such as polyuria and polydipsia, and biochemical findings of GS in our patient improved with oral potassium and magnesium supplementations in 20 months of follow-up period.

In a recent study, the variant c.602-16G $>A$ and the variant c. $2221 \mathrm{G}>\mathrm{A}$, both in heterozygosity, were detected in SLC12A3 gene, in a patient with GS and primary hyperparathyroidism. ${ }^{14}$ In another study, nine splice site mutations, including c.602-16G > A as a novel splice site mutation, were identified and predicted to lead to a frameshift and premature stopcodon. ${ }^{15}$ The variant c.602-16G $>A$ is described on the database of HGMD, as a disease-causing mutation. ${ }^{15}$ Furthermore, it is described in dbSNP and ExAC databases, and it's frequency is reported as $0.0016 \% .^{14}$ The functional analysis of mutations in SLC12A3 gene were performed for a limited number of mutations. Rego et al. ${ }^{14}$ reported that the variant c.602$16 \mathrm{G}>\mathrm{A}$ is considered of undetermined significance due to lack of functional analysis confirming the splicing effect.

Genetic heterogeneity that can be defined as mutations at two or more genetic loci producing the similar phenotypes should always be considered. On the other hand, the identification of a mutation in one gene does 
not always explain the clinical heterogeneity of the patients. However, it is still unknown in co-existence of two different diseases if there is not any common genetic susceptibility. Since this is the first case with homozygote mutations in both genes in the literature, the intersection of some disease characteristics has therefore led to proposals that this susceptibility exists if there would be more cases. Until that, for two disease entities in the same case with identified genetic alteration, it is unclear if this is coincidental, concomitant or causally related.

FMF is an inflammatory process and was recently linked to early atherosclerosis, whereas GS patients were shown to have blunted Angiotensin II signaling pathways and normal levels of C-reactive protein, serum amyloid A, vascular cell adhesion molecule, interleukin- 6 and tumor necrosis factor- $\alpha .^{16,17}$ Therefore, GS patients may not have an inflammatory phenotype such as hypertension or atherosclerosis. Our patient was treated successfully with enteral potassium and magnesium supplementation and under colchicine co-treatment, no metabolic disturbances have occurred.

Colchicine is a tricyclic alkaloid which is mainly metabolized in the liver and binds to free tubulin dimers which further disrupts microtubule polymerization. ${ }^{18}$ Electrolyte disturbances such as hyponatremia, hypokalemia, hypocalcemia, hypophosphatemia, hypomagnesemia and metabolic acidosis due to acute kidney injury, were reported in cases with colchicine intoxication. ${ }^{19,20}$ Huang et al. ${ }^{19}$ reported that colchicine overdose resulted in acute kidney injury by combining glucosuria, revealing that proximal tubular damage occurred in their case. Contrary to colchicine intoxications, there are no studies which report colchicine treatment to cause tubulopathies in the literature.

To the best of our knowledge, we report a rare association of FMF and GS and this is the first described case in an adolescent. Neither deterioration in FMF and GS symptoms, nor vascular inflammatory phenotype had developed in our patient. Nevertheless, the patient should be monitored for hypertension and atherosclerosis unlike the patients with GS only. Our patient responded well to colchicine at a dose of $1 \mathrm{mg}(0.03 \mathrm{mg} / \mathrm{kg})$ per day, but we could not associate better control of FMF attacks with co-existence of GS, due to lack of sufficient evidence.

The parents and sibling of our patient had normal serum electrolytes and parents were heterozygous for the c.602-16G > A mutation. Analysis of mutations in SLC12A3 gene were not performed for his sibling since there was no abnormality in serum electrolytes. This was a limitation for our case report.

In conclusion, we highlight the presence of inflammation related laboratory results and unexplained symptoms with polyuria/ polydipsia and metabolic disturbances in coexistence of GS with FMF.

\section{REFERENCES}

1. Jain G, Ong S, Warnock DG. Genetic disorders of potassium homeostasis. Semin Nephrol 2013; 33: 300-309.

2. Riveira-Munoz E, Chang Q, Bindels RJ, Devuyst O. Gitelman's syndrome: towards genotype-phenotype correlations? Pediatr Nephrol 2007; 22: 326-332.

3. Ben-Chetrit E, Levy M. Familial Mediterranean fever. Lancet 1998; 351: 659-664.

4. Erten S, Ceylan GG, Altunoğlu A. Concomitance of Gitelman syndrome and familial Mediterranean fever: a rare case presentation. Ren Fail 2012; 34: 13331334.

5. Livneh A, Langevitz P, Zemer D, et al. Criteria for the diagnosis of familial Mediterranean fever. Arthritis Rheum 1997; 40: 1879-1885.

6. Yalçinkaya F, Ozen S, Ozçakar ZB, et al. A new set of criteria for the diagnosis of familial Mediterranean fever in childhood. Rheumatology (Oxford) 2009; 48: 395-398.

7. Viering DHHM, de Baaij JHF, Walsh SB, Kleta R, Bockenhauer D. Genetic causes of hypomagnesemia, a clinical overview. Pediatr Nephrol 2017; 32: 1123 1135 .

8. Foglia PE, Bettinelli A, Tosetto C, et al. Cardiac work up in primary renal hypokalaemia-hypomagnesaemia (Gitelman syndrome). Nephrol Dial Transplant 2004; 19: 1398-1402.

9. Cruz DN, Shaer AJ, Bia MJ, Lifton RP, Simon DB; Yale Gitelman's and Bartter's Syndrome Collaborative Study Group. Gitelman's syndrome revisited: an evaluation of symptoms and health-related quality of life. Kidney Int 2001; 59: 710-717. 
10. Loffing J, Vallon V, Loffing-Cueni D, et al. Altered renal distal tubule structure and renal $\mathrm{Na}(+)$ and $\mathrm{Ca}(2+)$ handling in a mouse model for Gitelman's syndrome. J Am Soc Nephrol 2004; 15: 2276-2288.

11. Tunca M, Akar S, Onen F et al; Turkish FMF Study Group. Familial Mediterranean fever (FMF) in Turkey: results of a nationwide multicenter study. Medicine (Baltimore) 2005; 84: 1-11.

12. Özçakar ZB, Çakar N, Uncu N, Çelikel BA, Yalçınkaya F. Familial Mediterranean fever-associated diseases in children. QJM 2017; 110: 287-290.

13. Rigante D, Lopalco G, Tarantino G, Compagnone A, Fastiggi M, Cantarini L. Non-canonical manifestations of familial Mediterranean fever: a changing paradigm. Clin Rheumatol 2015; 34: 1503-1511.

14. Rego T, Fonseca F, Cerqueira R, Agapito A. Gitelman syndrome and primary hyperparathyroidism: a rare association. BMJ Case Rep 2018. doi: 10.1136/bcr2017-223663

15. Glaudemans B, Yntema HG, San-Cristobal P, et al. Novel NCC mutants and functional analysis in a new cohort of patients with Gitelman syndrome. Eur J Hum Genet 2012; 20: 263-270.
16. Peru H, Altun B, Doğan M, Kara F, Elmaci AM, Oran $B$. The evaluation of carotid intima-media thickness in children with familial Mediterranean fever. Clin Rheumatol 2008; 27: 689-694.

17. Caló LA, Davis PA, Pagnin E, Schiavo S, Semplicini A, Pessina AC. Linking inflammation and hypertension in humans: studies in Bartter's/Gitelman's syndrome patients. J Hum Hypertens 2008; 22: 223-225.

18. Slobodnick A, Shah B, Pillinger MH, Krasnokutsky S. Colchicine: old and new. Am J Med 2015; 128: 461470 .

19. Huang WH, Hsu CW, Yu CC. Colchicine overdoseinduced acute renal failure and electrolyte imbalance. Ren Fail 2007; 29: 367-370.

20. Milne ST, Meek PD. Fatal colchicine overdose: report of a case and review of the literature. Am J Emerg Med 1998; 16: 603-608. 